\title{
Variação espaço-temporal do ictioplâncton em praias estuarinas da baía da Babitonga, Santa Catarina, Brasil
}

\author{
José Maria Souza-Conceição ${ }^{1 *}$ \\ Henry Louis Spach ${ }^{2}$ \\ Micheli Duarte de Paula Costa ${ }^{3}$ \\ Daliana Bordin ${ }^{4}$ \\ ${ }^{1}$ EEB Alexandre Guilherme Figueredo - SED-SC \\ ${ }^{2}$ Centro de Estudos do Mar, Universidade Federal do Paraná, \\ Avenida Beira Mar, s/n, CEP 83255-000, Pontal do Sul - PR, Brasil \\ ${ }^{3}$ Laboratório de Ecologia do Ictioplâncton, Instituto de Oceanografia, Campus Carreiros \\ Caixa Postal 470, CEP 96201-900, Rio Grande - RS, Brasil \\ ${ }^{4}$ Universidade do Contestado \\ Rua Roberto Ehlke, 86, CEP 89460-000, Canoinhas - SC, Brasil \\ * Autor para correspondência \\ zzze.maria@yahoo.com.br
}

Submetido em 30/03/2012

Aceito para publicação em 22/01/2013

\section{Resumo}

No Brasil, as praias estuarinas são muito pouco estudadas em relação ao ictioplâncton. Nesse contexto, de agosto de 2005 a julho de 2006, foram realizadas coletas mensais, com uma rede de plâncton cônica, com $200 \mu \mathrm{m}$ de abertura de malha e $40 \mathrm{~cm}$ de diâmetro da boca, em sete praias estuarinas no setor polihalino da baía da Babitonga, Santa Catarina. Em cada praia foram obtidos dados relativos a temperatura, salinidade, oxigênio dissolvido, $\mathrm{pH}$, clorofila $a$, e biovolume zooplanctônico. No total foram coletados 54.384 ovos de peixes e 10.576 larvas de peixes, com abundância média geral de 3.114 ovos. $100 \mathrm{~m}^{-3} \mathrm{e} 607$ larvas. $100 \mathrm{~m}^{-3}$. Maior abundância de ovos ocorreu de outubro a março e maior abundância de larvas ocorreu de outubro a dezembro e entre fevereiro e abril. Entre as praias, nas intermediárias foi registrada maior abundância de ovos e nas mais externas (mais próximas da barra) foi registrada maior abundância de larvas. Ocorreu o predomínio de larvas das famílias Haemulidae, Engraulidae, Gobiidae, Sciaenidae, Blenniidae, Carangidae e Sparidae, a maioria presentes no período mais quente do ano. A análise das variáveis da coluna d'água, clorofila $a$, biovolume zooplanctônico e ictioplâncton revelou baixas correlações nos hábitats rasos estudados.

Palavras-chave: Abundância; Estuário; Larvas de peixes; Ovos de peixes; Sul do Brasil

\section{Abstract}

Spatio-temporal variation of ichthyoplankton in estuarine beaches at the Babitonga bay, Santa Catarina, Brazil. In Brazil, estuarine beaches are poorly studied with regard to ichthyoplankton. In this context, from August 2005 to July 2006, monthly collections were conducted, using conical plankton net with $200 \mu \mathrm{m}$ mesh size and $40 \mathrm{~cm}$ mouth diameter, at seven estuarine beaches in the polyhaline sector of Babitonga bay, Santa Catarina, Brazil. At each beach, data regarding temperature, salinity, dissolved oxygen, $\mathrm{pH}$, chlorophyll- $a$, and zooplankton volume were obtained. A total of 54,384 fish eggs and 10,576 fish larvae were collected, with a 
general mean abundance of 3,114 eggs. $100 \mathrm{~m}^{-3}$ and 607 larvae. $100 \mathrm{~m}^{-3}$. Higher abundance of eggs occurred from October to March and higher abundance of larvae occurred from October to December and between February and April. Among the beaches, higher abundance of eggs was recorded at the intermediate ones and higher abundance of larvae was recorded at the outermost ones (those closest to the estuary mouth). There was a predominance of larvae from the families Haemulidae, Engraulidae, Gobiidae, Sciaenidae, Blenniidae, Carangidae, and Sparidae, most of them found in the warmest period of the year. Analysis on the water column variables, chlorophyll- $a$, zooplankton volume and ichthyoplankton showed low correlations in the shallow habitats under study.

Key words: Abundance; Estuary; Fish eggs; Fish larvae; Southern Brazil

\section{Introdução}

A grande maioria dos peixes ósseos marinhos apresenta como fases iniciais do seu ciclo de vida, ovos e larvas planctônicos, os quais são integrantes do meroplâncton e estreitamente relacionados com a dinâmica do ecossistema (HOUDE; ZASTROW, 1993). As fases subsequentes, juvenis e adultos, apresentam maior capacidade de locomoção, o que possibilita a procura ativa de habitats e recursos alimentares. Estas fases, dependendo da espécie, podem estar associadas ao ecossistema pelágico, demersal ou bentônico (RÉ, 1999; RICHARDS, 2006).

O habitat estuarino é usado pelos peixes em parte ou ao longo de todo seu ciclo de vida, podendo também migrar através dos estuários entre áreas de alimentação e reprodução (ELLIOTT; HEMINGWAY, 2002). Também é grande a importância destas áreas durante o crescimento de espécies de peixes (BOEHLERT; MUNDY, 1988). A grande oferta de alimento, e consequente ganho no crescimento, assim como a proteção contra predadores, são determinantes em sua função de berçário (WEISBERG, 1996; RÖNNBÄCK, 1999). Na atualidade, mesmo com a crescente degradação dos estuários pela pressão antrópica, estes ecossistemas ainda mantêm expressiva abundância de peixes em diferentes estágios do ciclo de vida (HAJISAMAE; CHOU, 2003).

A avaliação do valor funcional dos habitats estuarinos como áreas de criação de peixes é, na maioria das vezes, derivada da amostragem de juvenis, existindo poucos estudos sobre as interações entre as larvas de peixes e as características físicas, químicas e biológicas que definem o habitat (ROUNTREE; ABLE, 1992). Entre os habitats estuarinos, as praias são um dos menos estudados (JACKSON et al., 2002), sendo desconhecidas, por exemplo, informações básicas sobre o ictioplâncton. No estado de Santa Catarina (Brasil), especialmente na baía da Babitonga (maior complexo estuarino catarinense), até o momento são inexistentes trabalhos com tal abordagem. Esta situação dificulta a avaliação de como as perturbações naturais ou induzidas pelo homem, que conduzam a perda ou a degradação ambiental, podem influenciar na sobrevivência das larvas. Neste sentido, o presente trabalho teve como objetivo estudar as variações espaço-temporais do ictioplâncton e sua interação com as variáveis bióticas e abióticas da coluna d'água no habitat de praias estuarinas na baía da Babitonga.

\section{Material e Métodos}

\section{Área de estudo}

A baía da Babitonga compõe o maior complexo estuarino do litoral de Santa Catarina, onde águas da plataforma adjacente se misturam com a drenagem continental, resultando em um caráter mixohalino, que o caracteriza como um estuário homogêneo (CREMER et al., 2006). Seu entorno é rodeado por florestas de mangue e importantes remanescentes de Mata Atlântica e restinga (KNIE, 2002), considerados fontes de nutrientes para a baía.

O clima da região, caracteristicamente, apresenta inverno e verão bem definidos (CREMER et al., 2006). A profundidade da baía da Babitonga atinge máximo de $28 \mathrm{~m}$ no canal principal de acesso ao porto de São Francisco do Sul e média de $6 \mathrm{~m}$, existindo áreas rasas que ficam expostas na maré baixa (IBAMA,1998). A variação da maré atinge uma amplitude de 2,3 m, com 
duração máxima aproximada de $6 \mathrm{~h}$, segundo dados da Capitania dos Portos para o Porto de São Francisco do Sul (MARMIL, 2012).

No setor ocidental a baía recebe aportes fluviais (principais rios: Palmital, Cubatão, Cachoeira e Parati), além de forte pressão antrópica diante das atividades econômicas dos 6 municípios de entorno; e no setor oriental recebe a influência do Oceano Atlântico (KNIE, 2002). O município de São Francisco do Sul, onde está inserida a maior parte da baía, possui área de $470 \mathrm{~km}^{2}$, compreendendo toda a Ilha de São Francisco e parte do continente na porção central. No sul da ilha encontrase o Canal do Linguado, o qual foi obstruído por aterro para a passagem ferroviária e rodoviária (CREMER et al., 2006). Além disso, a cidade mantém atividades portuárias regulares e recebe muitos turistas ao longo do ano, o que sugere a necessidade de um monitoramento constante da região.

\section{Coleta de dados}

De agosto de 2005 a julho de 2006 foram realizadas coletas mensais na preamar de quadratura em sete praias estuarinas, distribuídas no setor polihalino da baía da Babitonga (Figura 1).

Para a obtenção das amostras de ictioplâncton, foram realizados, a bordo de um barco com motor de $30 \mathrm{HP}$, dois arrastos oblíquos em trajetória circular, na área adjacente de cada ponto amostral utilizando uma rede de plâncton cônica, com $200 \mu \mathrm{m}$ de abertura de malha, $40 \mathrm{~cm}$ de diâmetro de boca e equipada com um fluxômetro para estimar o volume de água filtrado. O modelo de rede foi escolhido para abranger os estágios de desenvolvimento das menores classes de tamanho dentro do ictioplâncton, reduzindo o escape e a extrusão (CHUTE; TURNER, 2001).

FIGURA 1: Localização da baía da Babitonga e dos sete pontos de amostragem em praias estuarinas.

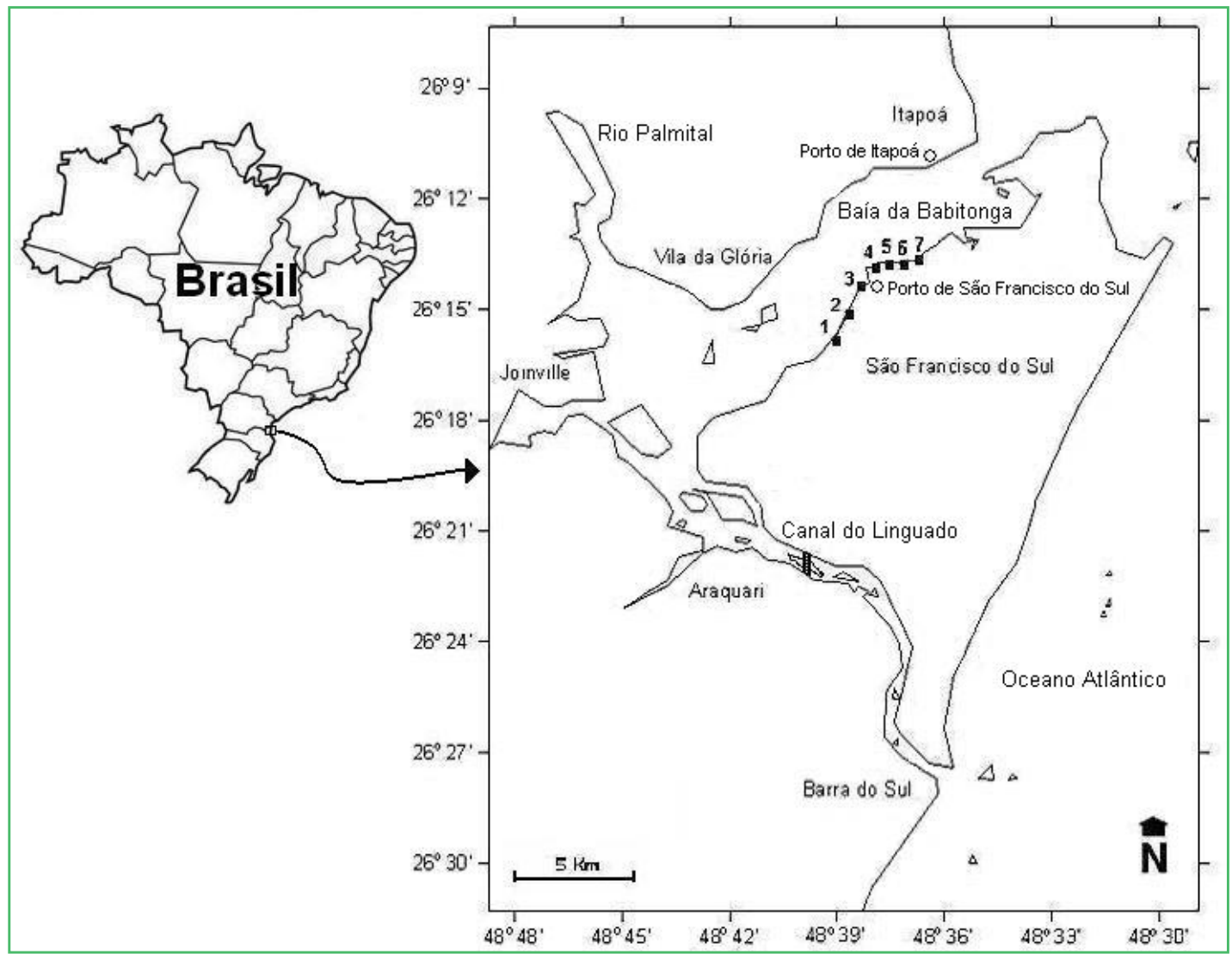


No momento de cada coleta e antes do primeiro arrasto foram obtidos in situ dados dos parâmetros físicos e químicos de temperatura $\left({ }^{\circ} \mathrm{C}\right)$, salinidade, pH e oxigênio dissolvido (mg. $\mathrm{L}^{-1}$ ) utilizando-se um multianalisador HORIBA modelo U-10 em subsuperfície. Para a clorofila $a$, amostras de água subsuperficial foram coletadas com garrafa modelo Van Dorn e retiradas alíquotas de 100 a $150 \mathrm{~mL}$, as quais foram filtradas a bordo com um sistema de filtração e filtros (Whatman) de fibra de vidro com $25 \mathrm{~mm}$ de diâmetro e 1,2 $\mu \mathrm{m}$ de abertura de poro (GF/C). Após a filtragem os filtros foram acondicionados em papel alumínio para minimizar a interferência da luz, etiquetados e mantidos em gelo para posterior análise no laboratório. Os pigmentos foram extraídos com acetona $90 \%$ durante $24 \mathrm{~h}$ no escuro a $-12^{\circ} \mathrm{C}$. Depois deste período, o material sobrenadante foi colocado em cubetas ópticas de $1 \mathrm{~cm}^{3}$ e realizadas as leituras em fluorímetro TURNER, modelo TD-700 (MACHADO et al., 1997). No laboratório, o biovolume de zooplâncton foi determinado por meio do método volumétrico, em mililitros, e, posteriormente, calculadas as abundâncias $\left(\mathrm{mL} .100 \mathrm{~m}^{-3}\right)$. Os dados abióticos da coluna d'água e de clorofila $a$ foram utilizados para analisar a influência ambiental na variação das abundâncias do ictioplâncton e do biovolume.

Ovos e larvas de peixes foram triados das amostras de plâncton e, então, calculadas as abundâncias (ovos. $100 \mathrm{~m}^{-3}$ e larvas. $100 \mathrm{~m}^{-3}$ ). As larvas foram identificadas ao menor nível taxonômico possível de acordo com referências bibliográficas especializadas (FAHAY, 1983; LEIS; RENNIS, 1983; LEIS; TRNSKI, 1989; MOSER, 1996; KAWAGUCHI et al., 1999; RÉ, 1999; RICHARDS, 2006).

\section{Processamento dos dados}

Para identificar variações temporais e espaciais na temperatura, salinidade, $\mathrm{pH}$, oxigênio dissolvido da água, concentração de clorofila $a$, biovolume de zooplâncton e nas abundâncias ( $\log _{10}+1$ transformadas) de ovos e de larvas, foi utilizada a anova unifatorial. Todos os dados foram testados quanto à homogeneidade da variância (Teste de Bartellet) e normalidade (Teste de Kolmogorov-Smirnov). Onde ocorreram diferenças significativas $(\mathrm{p}<0,01$ e $\mathrm{p}<0,05)$, aplicou-se o teste $a$ posteriori de Tukey (SOKAL; ROHLF, 1995).

Para examinar até que ponto os dados físicos, químicos e de clorofila $a$, considerados individualmente e combinados, podem explicar os padrões de abundância de ovos e larvas e do biovolume, comparou-se através do coeficiente de correlação harmônico ponderado "Spearman rank" as matrizes de dissimilaridades dos dados bióticos e abióticos (BIOENV) (CLARKE; WARWICK, 2001).

\section{Resultados}

A temperatura não variou significamente entre os pontos amostrais, porém entre meses as diferenças foram significativas, com maiores temperaturas em janeiro, fevereiro e março e menores em agosto, setembro e outubro (Figura 2; Tabela 1). Entre as praias não foram observadas diferenças estatísticas na salinidade. Temporalmente a salinidade foi significativamente maior em maio em relação aos demais meses, com exceção de outubro, e maior nos meses de outubro, novembro e agosto em comparação a fevereiro, março e julho (Figura 2; Tabela 1). O pH ocorreu com homogeneidade espacial, com o pH de junho sendo em média menor que os dos outros meses, excetuando-se outubro e julho (Figura 2; Tabela 1). $\mathrm{O}$ oxigênio dissolvido foi maior na praia 1 e em relação a praia 7, e ao longo do ano foi maior em janeiro, abril, junho e julho em relação a novembro (Figura 2; Tabela 1).

Espacialmente o biovolume foi homogêneo, enquanto que a clorofila $a$ foi maior nas praias 1 e 2, em relação a praia 7 (Figura 3; Tabela 1). Ao longo do ano a abundância de biovolume foi maior em novembro em comparação aos demais meses, excetuando-se outubro, e foi maior também nos meses de junho, julho e outubro em relação a agosto, setembro, fevereiro e março. As concentrações de clorofila $a$ foram mais elevadas em janeiro e março em relação a setembro, novembro, abril, junho e julho, e mais elevadas também nos meses de outubro, dezembro e fevereiro, quando comparados com setembro, novembro e julho (Figura 3; Tabela 1). 
FIGURA 2: Variação espaço-temporal (médias \pm erro padrão) dos parâmetros de temperatura, salinidade, pH e oxigênio dissolvido nas praias estuarinas, baía da Babitonga (SC).
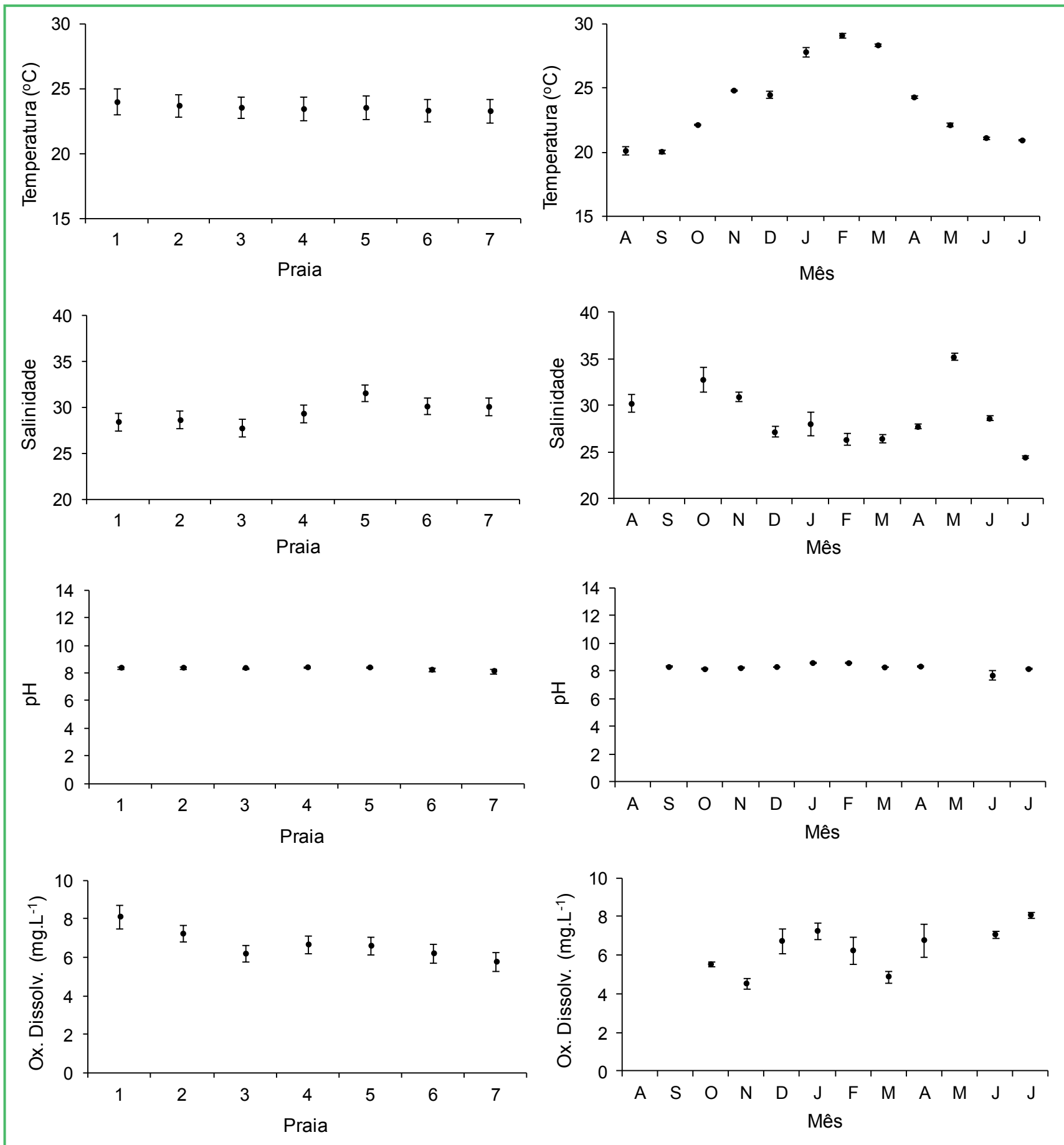
No total foram coletados 54.384 ovos e 10.576 larvas de peixes, abundância média geral de 3.114 ovos. $100 \mathrm{~m}^{-3}$ e 607 larvas. $100 \mathrm{~m}^{-3}$, sendo observado durante a triagem grande participação de larvas vitelinicas (em geral $>50 \%$ ). Em média o número de ovos foi significativamente diferente entre as praias, com médias maiores nas praias 1, 2 e 3 em relação a praia 7 , não existindo diferenças significativas entre as demais praias (Figura 4; Tabela 1). Diferença significativa também foi observada entre as médias mensais do número de ovos, com média maior em novembro. Entre as praias não foram observadas diferenças significativas no número médio de larvas. Entre os meses o número médio de larvas apresentou diferenças significativas, sendo maior em novembro e menor em junho (Figura 4; Tabela 1).

TABELA 1: Resultado da Análise de Variância avaliando o efeito do mês e ponto de coleta sobre a temperatura, salinidade, $\mathrm{pH}$, oxigênio dissolvido, biovolume, clorofila $a$, número de ovos e número de larvas (NS diferença não significativa).

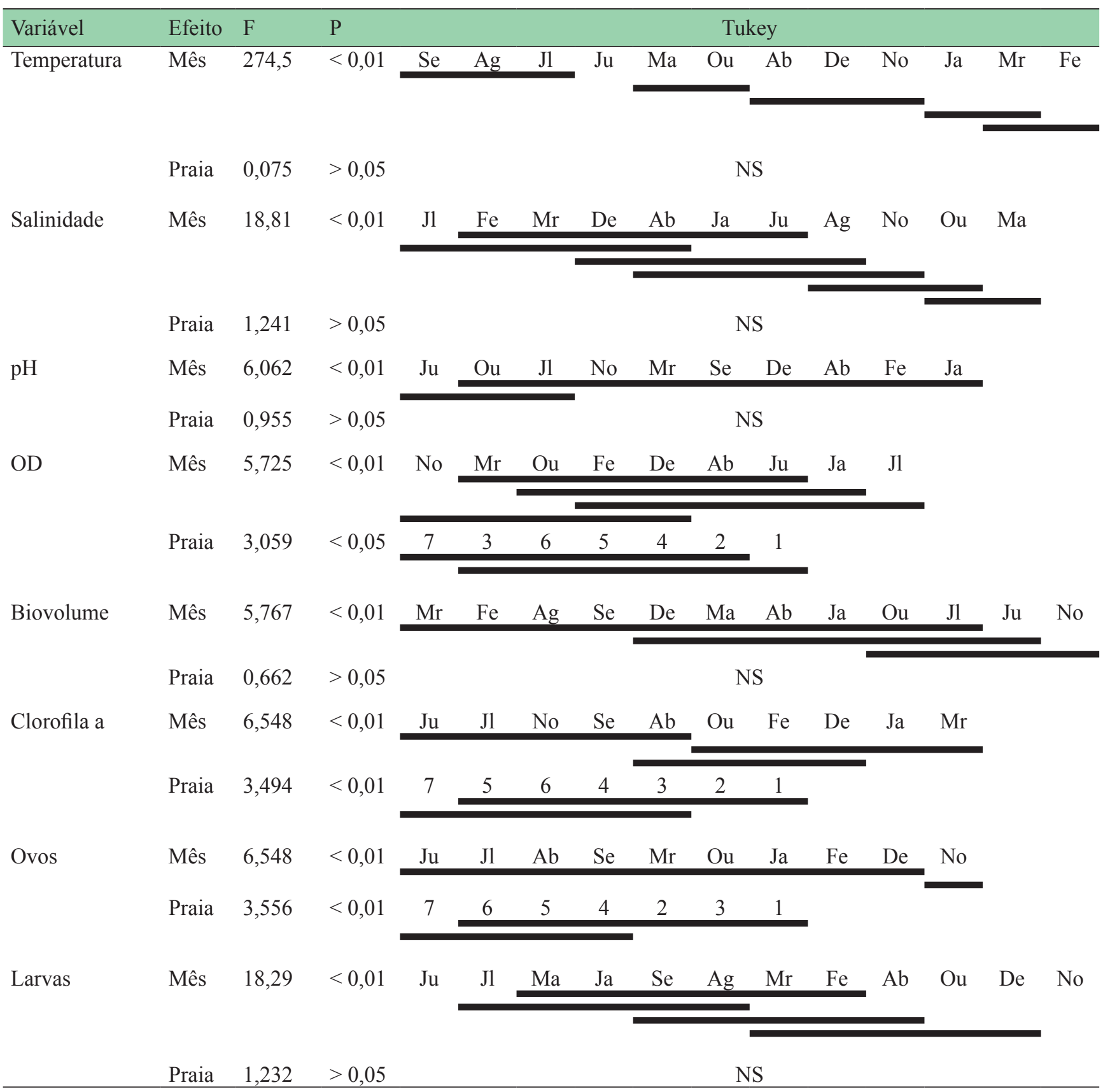


Na identificação foram registradas larvas pertencentes a 39 táxons, sendo 14 famílias, 13 gêneros e 12 espécies. Ocorreu o predominio de Haemulidade, Engraulidae, Gobiidae, Sciaenidae, Blenniidae, Carangidae e Sparidae, em sua maioria presentes no período mais quente do ano. Entretanto, apenas Blennidae, Gobiidae, Haemulidae e Sciaenidae estiveram presentes o ano todo na área.
Larvas de Achiridae ocorreram de setembro a março, Sparidae de agosto a dezembro, Engraulidae de outubro a maio (com excessão de abril), enquanto que Carangidae de agosto a abril. Ocorreram padrões de distribuição temporal específicos para as larvas das demais famílias, normalmente indicando sazonalidade em sua ocorrência (Tabela 2).

FIGURA 3: Variação espaço-temporal (médias \pm erro padrão) da abundância do biovolume (zooplâncton) e das concentrações de clorofila $a$ nas praias estuarinas, baía da Babitonga (SC).
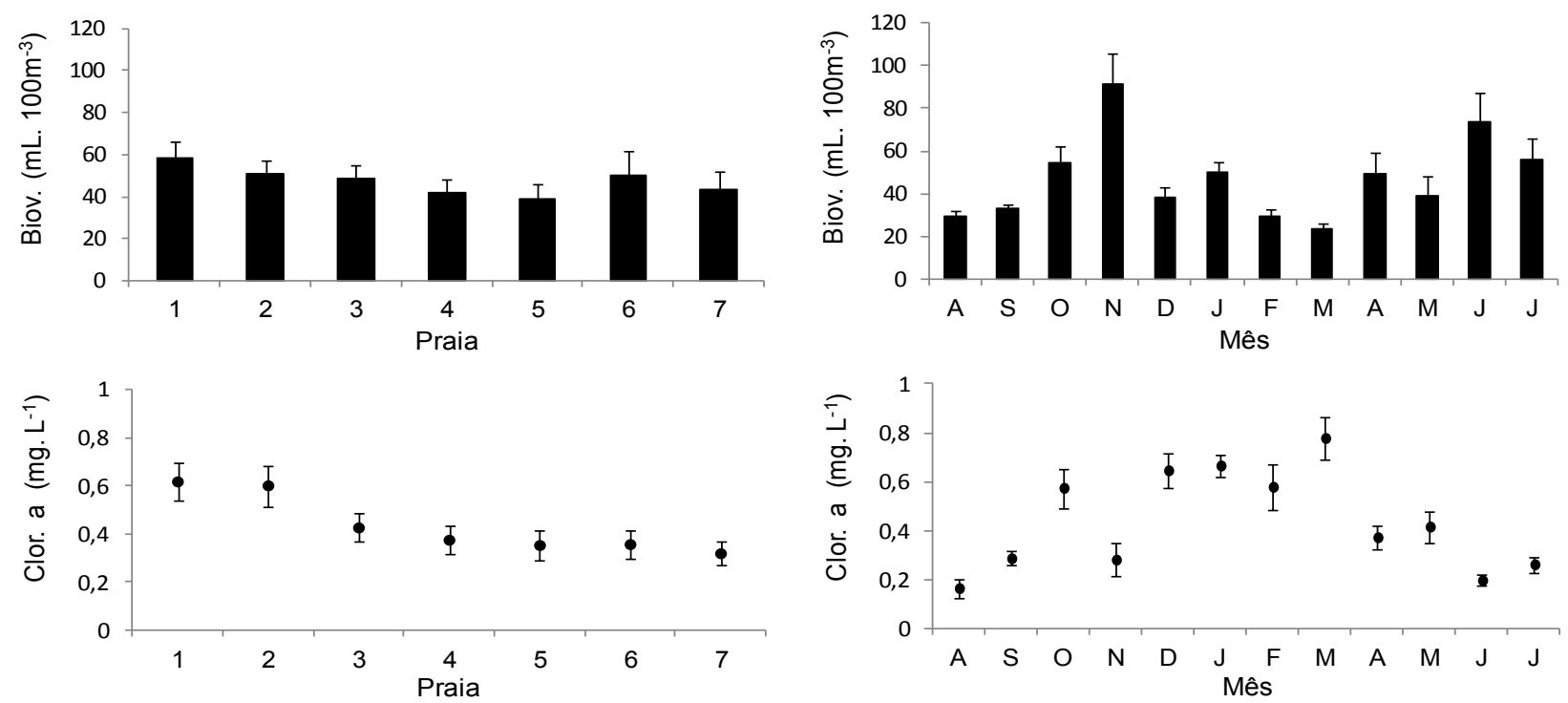

FIGURA 4 : Variação espaço-temporal das abundâncias (médias \pm erro padrão) de ovos e larvas de peixes nas praias estuarinas, baía da Babitonga (SC).

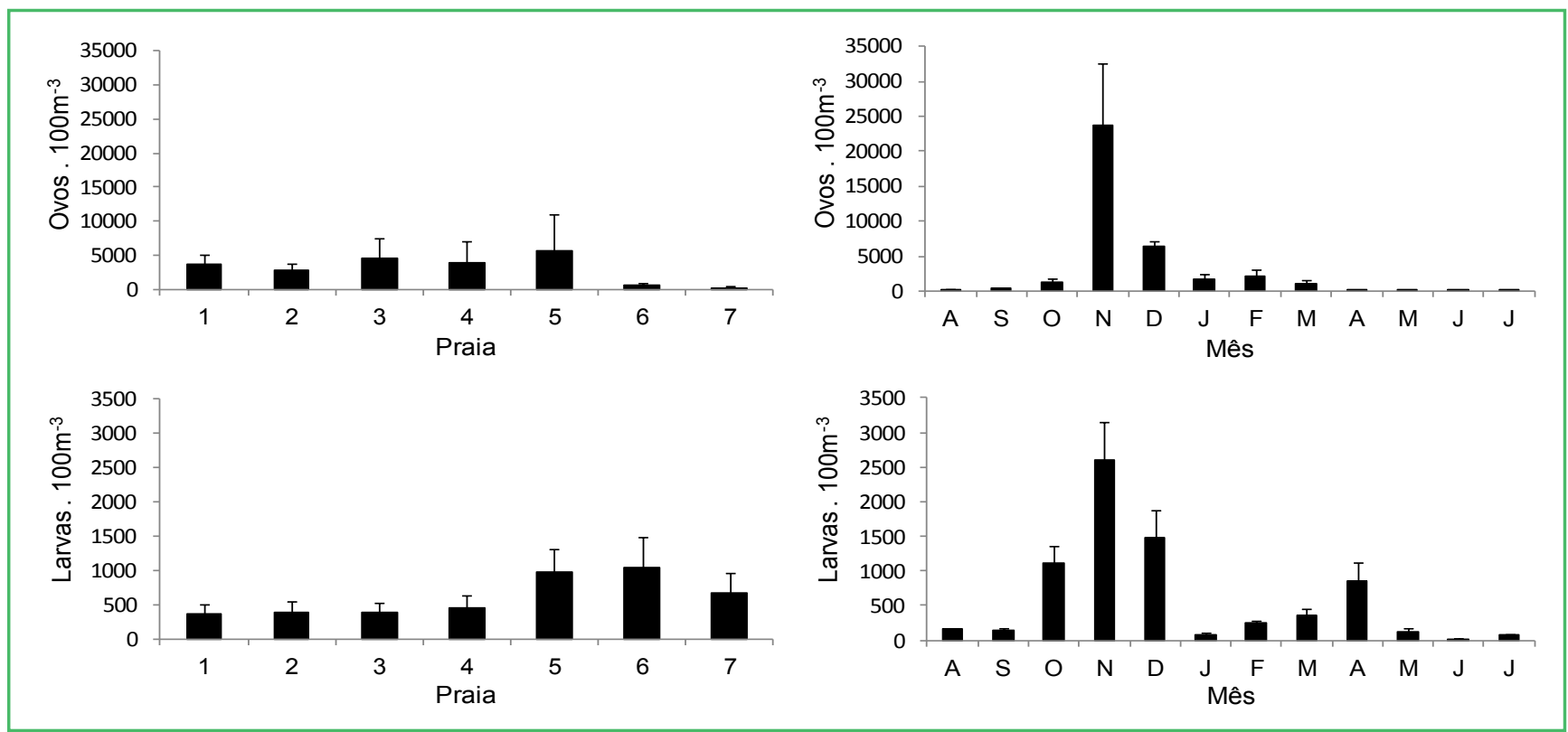


Uma distribuição similar ao longo da área estudada, porém com abundâncias diferentes, ocorreu com as larvas de Blenniidae, Sparidae, Carangidae, Gobiesocidae, Achiridae, Serranidae Engraulidae e Tetraodontidae. As abundâncias de larvas foram maiores nas praias 4, 5, 6 e 7 para Haemulidae, 1, 2 e 3 para Sciaenidae e 5, 6 e 7 para Gobiidae. As larvas identificadas além do nível de família corresponderam a 25 táxons, registradas principalmente em novembro e dezembro. A ocorrência de abundâncias mais expressivas destas larvas foi observada nas praias 2, 3, 4, 5 e 6, com grande participação de Citharichthys sp., Orthopristis ruber, Oligoplites sp., Diplectrum radiale, Diplodus argenteus e Omobranchus punctatus. Ocorrência restrita nas praias mais internas foi observada para Sphyraena sp. Entretanto, alguns registros foram exclusivos a uma praia, como por exemplo, Mugil curema, Cynoscion sp., Archosargus rhomboidalis, entre outros (Tabela 2).

TABELA 2 : Lista taxonômica e variação espaço-temporal das abundâncias médias (larvas. $100 \mathrm{~m}^{-3}$ ) registradas nas praias estuarinas, baía da Babitonga (SC, Brasil).

\begin{tabular}{|c|c|c|c|c|c|c|c|c|c|c|c|c|c|c|c|c|c|c|c|}
\hline \multirow{2}{*}{ Táxons } & \multicolumn{7}{|c|}{ Praias } & \multicolumn{12}{|c|}{ Meses } \\
\hline & 1 & 2 & 3 & 4 & 5 & 6 & 7 & $\mathbf{A}$ & $\mathbf{S}$ & 0 & $\mathbf{N}$ & D & $\mathbf{J}$ & $\mathbf{F}$ & M & A & M & $\mathbf{J}$ & $\mathbf{J}$ \\
\hline Achiridae & 19,4 & 8,3 & 13,0 & 12,6 & 11,6 & 9,8 & 13,9 & - & 6,4 & 11,7 & 17,6 & 12,4 & 8,8 & 29,1 & 7,7 & - & - & - & - \\
\hline Blenniidae & 25,6 & 21,8 & 48,3 & 31,3 & 53,2 & 41,2 & 32,4 & 53,8 & 24,0 & 42,3 & 55,1 & 76,4 & 10,4 & 12,7 & 11,4 & 28,5 & 42,6 & 23,0 & 51,9 \\
\hline Omobranchus punctatus & 7,2 & 18,5 & 12,0 & 10,6 & 12,5 & 8,0 & - & 5,8 & - & 11,5 & 12,6 & 14,6 & - & 8,6 & 5,0 & - & - & - & - \\
\hline Scartella cristata & - & 17,9 & - & - & 7,1 & - & - & 17,9 & - & - & - & 7,1 & - & - & - & - & - & - & - \\
\hline Carangidae & 29,0 & 16,4 & 21,7 & 42,7 & 52,5 & 15,2 & 24,5 & 8,4 & 6,7 & 21,3 & 27,1 & 75,9 & 5,1 & 18,4 & 21,0 & 8,5 & - & - & - \\
\hline Chloroscombrus chrysurus & - & - & - & - & - & 11,8 & - & - & - & - & - & - & 11,8 & - & - & - & - & - & - \\
\hline Oligoplites sp. & - & - & 7,8 & 9,8 & - & 15,0 & 7,4 & - & - & - & - & 8,8 & 15,0 & 8,6 & - & - & - & - & - \\
\hline Seriola sp. & - & - & 9,8 & - & - & - & - & - & - & - & 9,8 & - & - & - & - & - & - & - & - \\
\hline Trachurus lathami & - & - & - & 7,0 & - & 11,6 & - & - & - & 12,5 & - & 7,0 & - & - & - & - & - & - & - \\
\hline Engraulidae & 526,3 & 395,1 & 349,4 & 212,1 & 524,4 & 1212,0 & 826,0 & - & - & 408,4 & 1868,6 & 441,0 & 10,4 & 15,0 & 12,6 & - & 7,0 & - & - \\
\hline Eucinostomus sp. & - & 5,4 & - & - & - & - & 7,8 & - & - & - & - & - & 10,8 & - & 5,1 & - & - & - & - \\
\hline Gobiesocidae & - & 10,7 & 7,8 & 20,9 & 16,0 & 6,4 & 7,5 & - & 6,3 & 10,0 & 16,0 & 20,9 & - & - & - & - & 7,5 & - & 6,5 \\
\hline Gobiidae & 44,3 & 75,4 & 54,0 & 48,7 & 115,8 & 268,5 & 115,6 & 6,8 & 17,9 & 56,7 & 97,1 & 253,6 & 52,3 & 81,6 & 75,3 & 227,6 & 29,5 & 8,6 & 13,6 \\
\hline Haemulidae & 52,8 & 33,4 & 101,3 & 299,1 & 510,8 & 335,8 & 184,8 & 104,7 & 110,2 & 358,8 & 258,8 & 563,1 & 24,4 & 96,5 & 204,1 & 617,2 & 89,2 & 7,8 & 22,4 \\
\hline Orthopristis ruber & - & - & - & 7,0 & 34,5 & 34,9 & 10,0 & - & - & - & 31,8 & 15,5 & - & - & 4,8 & - & - & - & - \\
\hline Mugil curema & - & 9,0 & - & - & - & - & - & - & - & - & - & - & - & 9,0 & - & - & - & - & - \\
\hline Mugil sp. & - & - & 10,2 & 6,3 & - & - & - & 6,3 & - & - & - & - & - & - & 10,2 & - & - & - & - \\
\hline Paralichthyidae & 21,1 & - & 8,2 & 13,4 & 17,0 & 8,9 & 9,5 & - & - & 13,4 & - & 28,4 & 7,5 & - & 5,4 & 9,5 & - & - & 10,3 \\
\hline Citharichthys sp. & 9,5 & 5,6 & 19,6 & 6,4 & 6,8 & - & 11,4 & 15,3 & 6,7 & - & 13,4 & 7,4 & - & - & - & - & - & - & - \\
\hline Etropus sp. & - & - & - & - & - & - & 7,4 & - & - & - & - & 7,4 & - & - & - & - & - & - & - \\
\hline Pomacentridae & - & - & - & 7,0 & - & - & - & - & - & - & - & 7,0 & - & - & - & - & - & - & - \\
\hline Sciaenidae & 159,5 & 156,0 & 78,7 & 52,5 & 56,9 & 69,8 & 35,8 & 33,9 & 6,1 & 200,8 & 151,6 & 73,3 & 14,2 & 73,8 & 48,8 & 9,0 & 7,1 & - & 9,8 \\
\hline Cynoscion sp. & 7,7 & - & - & - & - & - & - & - & - & - & - & 7,7 & - & - & - & - & - & - & - \\
\hline Menticirrhus sp. & - & - & - & 10,5 & - & - & - & - & - & - & 10,5 & - & - & - & - & - & - & - & - \\
\hline Pogonias cromis & - & 5,4 & - & - & - & - & - & - & - & - & - & - & - & - & 5,4 & - & - & - & - \\
\hline Umbrina sp. & 7,7 & - & - & 10,7 & 7,8 & - & - & - & - & - & 10,7 & 7,7 & 7,8 & - & - & - & - & - & - \\
\hline Serranidae & 7,7 & 5,4 & - & - & 6,6 & - & - & - & 6,7 & - & - & 6,6 & 7,7 & - & 5,4 & - & - & - & - \\
\hline Diplectrum radiale & - & - & - & 7,0 & 79,7 & - & - & - & - & - & 79,7 & 7,0 & - & - & - & - & - & - & - \\
\hline Serranus sp. & - & - & - & - & - & 7,5 & - & - & - & - & - & - & 7,5 & - & - & - & - & - & - \\
\hline Sparidae & 25,4 & 23,5 & 22,4 & 16,1 & 46,2 & 20,6 & 11,0 & 20,6 & 19,5 & 33,9 & 24,8 & 6,8 & - & - & - & - & - & - & - \\
\hline Archosargus rhomboidalis & - & - & - & 31,5 & - & - & - & - & - & - & 31,5 & - & - & - & - & - & - & - & - \\
\hline Calamus sp. & - & - & 7,8 & - & - & - & - & - & - & - & - & 7,8 & - & - & - & - & - & - & - \\
\hline Diplodus argenteus & - & - & - & - & 79,7 & - & - & - & - & - & 79,7 & - & - & - & - & - & - & - & - \\
\hline Sphyraena sp. & 21,7 & 6,2 & 6,3 & - & - & - & - & - & 11,4 & - & - & - & - & - & - & - & - & - & - \\
\hline Peprilus paru & - & 5,4 & - & - & 5,7 & - & - & - & - & - & - & - & - & - & 5,5 & - & - & - & - \\
\hline Syngnathidae & - & - & - & - & - & - & 7,5 & - & - & - & - & - & - & - & - & - & 7,5 & - & - \\
\hline Syngnathus rousseau & - & - & - & 7,0 & - & - & - & - & - & - & - & 7,0 & - & - & - & - & - & - & - \\
\hline Tetraodontidae & 10,0 & - & 11,2 & 10,7 & 14,8 & 13,3 & 13,7 & - & - & 14,3 & 16,8 & 9,5 & - & - & - & - & - & - & - \\
\hline Sphoeroides sp. & - & - & 7,8 & - & - & - & - & - & - & - & - & 7,8 & - & - & - & - & - & - & - \\
\hline
\end{tabular}


A provável influência das variáveis ambientais sobre o padrão de ocorrência de ovos e larvas de peixes e do biovolume foi examinada através do coeficiente de correlação de Sperman. Os resultados revelaram baixas correlações entre os parâmetros abióticos e bióticos analisados (Tabela 3).

TABELA 3: Resultados mais significativos de correlação (BIOENV) indicando a influência da temperatura da água $(\mathrm{T})$, salinidade $(\mathrm{S}), \mathrm{pH}$ (P), oxigênio dissolvido (O) e clorofila $a(\mathrm{C})$ sobre o padrão de ocorrência do biovolume e de ovos e larvas de peixe. Os valores são do coeficiente de correlação de Spearman para variável isolada e cada combinação.

\begin{tabular}{cc}
\hline Variável ou Combinação & Coeficiente \\
\hline POC & 0,186 \\
TOC & 0,165 \\
OC & 0,179 \\
T & 0,153 \\
TPOC & 0,173 \\
PC & 0,149 \\
TC & 0,167 \\
POSC & 0,139 \\
TPC & 0,165 \\
TPOSC & 0,133 \\
\hline
\end{tabular}

\section{Discussão}

As abundâncias do ictioplâncton registradas foram elevadas quando comparadas a outros estudos em áreas estuarinas no sul do Brasil (MUELBERT; WEISS, 1991; MACEDO-SOARES et al., 2009). As variações espaciais das abundâncias de larvas de peixes revelaram maior abundância nas praias mais externas da área amostrada, o que sugere a presença de condições abióticas e bióticas mais favoráveis a sua sobrevivência nestes locais, ocorrendo então, retenção larval. A expressiva presença de larvas vitelínicas é um indicativo de proximidade das áreas de desova. Por outro lado, as abundâncias mais elevadas de ovos em determinadas praias refletem o resultado de padrões de circulação da baía da Babitonga pois, por sua passividade,os ovos são carreados até estes locais. Os ovos também indicam sítios de desova ou proximidade de onde ocorreu sua liberação. Outros trabalhos confirmam as afirmações acima para larvas e ovos (BOEHLERT; MUNDY, 1988;
McLUSKY, 1994; CHURCHILL et al., 1999; RÉ, 1999; HAKALA et al., 2003; SOUZA-CONCEIÇÃO et al., 2005 ; VÉLEZ et al., 2005).

Os habitats estuarinos podem funcionar como áreas de desova, de criação, de alimentação e como vias de migrações diádromas, sendo que o indicativo da função de área de desova é a abundância de ovos (ELLIOTT; HEMINGWAY, 2002). Ao longo deste estudo ocorreram abundâncias de ovos elevadas, principalmente em novembro e dezembro, revelando sazonalidade bastante definida quanto ao principal período de desovas nas praias estuarinas e, consequentemente, em toda a área da baía, corroborando com Costa e Souza-Conceição (2009). Este período de desovas é marcado pela presença de um conjunto de ovos de vários táxons que ocorrem na área, o que inclui aqueles identificados entre as larvas. Johannes (1978) descreve que em geral a atividade reprodutiva dos peixes marinhos costeiros estende-se sobre considerável porção do período anual, existindo certos períodos do ano em que grande número de espécies e de seus espécimes reproduz, sendo então denominados como picos coletivos de desovas. Entretanto, na utilização do ictioplâncton para um entendimento refinado destes picos coletivos de desovas, assim como de sua dinâmica espaço-temporal na área estudada, seria necessária a identificação específica dos ovos, tarefa muito difícil devido à escassez de descrições desta fase inicial das espécies presentes na costa brasileira.

As maiores abundâncias de ovos e larvas de peixes observadas no presente estudo (outubro a dezembro) confirmam a alta atividade reprodutiva na área, fortemente ajustada aos ciclos naturais de produção e antecedendo, de acordo com IBAMA (1998) e Cremer et al. (2006), as maiores abundâncias de juvenis. Neste caso, comprova-se o uso da área ao longo da sequência natural do ciclo de vida destes organismos. Thayer et al. (1999) e Ikejima et al. (2003) registraram a mesma sequência temporal para ovos, larvas e, nestes trabalhos, juvenis, com sazonalidade ajustada às épocas mais produtivas, o que corrobora com Lasker (1984). A sazonalidade da desova tem estreita relação ao requerimento das larvas por uma quantidade adequada de plâncton na faixa de tamanho que possa ser ingerido, e táxons com período de desova de maior amplitude 
diminuem a chance de expor toda a prole a um período pobre de alimento (WOOTTON, 1992). Na baía da Babitonga ocorreu ajuste sazonal entre a elevação das abundâncias de ovos e larvas de peixes, e dos demais componentes planctônicos, avaliados por meio do biovolume do zooplâncton e da clorofila $a$.

As praias estuarinas da baía da Babitonga desempenham importante papel para os primeiros estágios do ciclo de vida de muitos táxons de peixes costeiros e estuarinos, servindo como área de berçário. A composição do ictioplâncton foi marcada pela elevada abundância de Haemulidade, Engraulidae, Gobiidae, Sciaenidae, Blenniidae, Carangidae e Sparidae. Outros habitats desta baía têm assembleia similar conforme Costa e Souza-Conceição (2009), assim como outros estuários no Brasil (MUELBERT; WEISS, 1991; BARLETTA-BERGAN et al., 2002; BONECKER et al., 2009).

Dados de composição e abundância do ictioplâncton auxiliam na elucidação de padrões locais de distribuição e desova de espécies residentes e não residentes, assim como para determinar a importância de uma área como berçário (CHUTE ; TURNER, 2001). Garcia e Vieira (2001) descrevem que muitas espécies de peixes permanecem na região estuarina apenas por um curto período de tempo, enquanto outras completam todo o seu ciclo de vida no estuário. As praias estudadas na baía da Babitonga também possuem táxons que tipicamente permanecem por um período de tempo no estuário, como por exemplo, representantes de Carangidae, Mugilidae e Sciaenidae. Embora neste estudo não seja possível determinar a origem das larvas (estuarina ou marinha), é possível afirmar que, devido à expressiva presença de vitelínicas observada na triagem, grande parte eclodiu nas proximidades das praias, área de canal ou plataforma continental interna adjacente à baía. Segundo Fisher et al. (2004), as espécies marinhas visitantes ocasionais, como, por exemplo, representantes de Carangidae, representam um grupo numeroso que aparece irregularmente nas águas estuarinas. Os taxa Peprilus paru e Cynoscion sp. também são exemplos de larvas de origem marinha (SINQUE; MUELBERT, 1998) e que constam entre as identificações nas praias estuarinas.
Grande parte dos peixes coletados em subambientes de baías estuarinas, como as praias, estão em estágios iniciais de desenvolvimento, sendo que muitos destes são representantes de estoques comerciais nas águas de influência do estuário (LUGENDO et al., 2007). Entre os táxons registrados observou-se expressiva participação de representantes rotineiros na pesca artesanal de subsistência da área de estudo e outros representantes de estoques pesqueiros ao nível industrial na região Sul do Brasil, como por exemplo, Archosargus rhomboidalis, Carangidae, Chloroscombrus chrysurus, Citharichthys sp., Menticirrhus sp., Mugil curema, Mugil sp., Oligoplites sp., Peprilus paru, Pogonias cromis, Sciaenidae, Serranidae, Sphoeroides sp. e Trachurus lathami. A pesca artesanal de subsistência possui grande importância sócio-econômica no complexo estuarino da baía da Babitonga (CREMER et al., 2006), sendo estimado que cerca de mil famílias dependem diretamente da atividade de pescadores nas áreas interna e adjacente (plataforma continental) do estuário (IBAMA, 1998). No estuário da Laguna dos Patos (sul do Brasil) a pesca artesanal também possui grande importância, onde, por exemplo, representantes de Sciaenidae estão entre os mais abundantes tanto como adultos quanto ictioplâncton (GARCIA; VIEIRA, 1997; SINQUE; MUELBERT, 1998).

Apesar dos resultados obtidos na análise BIOENV revelarem baixa correlação entre o ictioplâncton e as variáveis abióticas e bióticas da coluna d'água, os dados do ictioplâncton indicaram condições ambientais favoráveis ao aumento das abundâncias de ovos e larvas condizentes com meses de primavera e de transição entre verão/outono, os quais coincidem com os principais períodos reprodutivos na área (IBAMA, 1998; CREMER et al., 2006; COSTA; SOUZA-CONCEIÇÃO, 2009). Maiores abundâncias de ictioplâncton nas praias estudadas, coincidem espacialmente, com menores concentrações de clorofila $a$, a qual aumentou em direção às praias mais internas. As abundâncias de ovos foram maiores nas condições ambientais das praias intermediárias, enquanto as de larvas naquelas mais externas. Por outro lado, a dinâmica temporal entre a elevação da abundância de ovos e larvas de peixes, da clorofila $a$ e do biovolume de zooplâncton observada no período que antecede o pico de temperatura, revelou 
antecipação das desovas para que a prole, ao eclodir, disponha de mais recursos alimentares. Exemplos em estuários da influência nos diferentes estágios da vida dos peixes das mesmas variáveis abióticas e bióticas analisadas neste estudo são encontrados em McLusky (1994), Jobling (1996), Lorenz (1999), Claireaux e Audet (2000) e Tsuzuki et al. (2000).

Um aspecto importante a considerar é que as condições ambientais correlatas espacialmente com maiores abundâncias de ovos podem, na realidade, ser secundárias, ou seja, podem simplesmente refletir as características da principal área no estuário (intermediária) para concentração destes, resultante da circulação agindo sobre sua deriva passiva. Esta hipótese reitera a necessidade de estudos sobre a interação da circulação na área com o transporte do ictioplâncton. Jackson et al. (2002) descrevem que nas pesquisas realizadas em praias estuarinas as interações entre os organismos e a dinâmica ambiental foram pobremente abordadas e raramente especificadas.

Beck et al. (2001; 2003) e Elliott e Hemingway (2002) descrevem uma série de fontes de alterações, ameaças, possibilidades de manejo e importâncias de áreas de criação estuarinas. A partir do presente trabalho na baía da Babitonga, destacam-se os sete subambientes de praia estuarina estudados e o cenário de incertezas que existe sobre sua conservação. A baía da Babitonga possui elevada importância ecológica, econômica e social, porém já apresenta claros sinais de degradação pela forte pressão antrópica existente (IBAMA, 1998). O registro neste estudo de $O$. punctatus com abundâncias e ocorrência espaço-temporal expressivas chama a atenção em termos ambientais de como é duvidosa a conservação deste complexo estuarino brasileiro. Omobranchus punctatus foi capturado na área em estudos anteriores no estágio adulto, sendo considerada uma espécie exótica, possivelmente com introdução mediada por navios (GERHARDINGER et al., 2006; FREITAS; VELASTIN, 2010). No ictioplâncton, a mesma foi registrada na maior parte deste estuário em abundâncias relativamente elevadas (COSTA et al., 2011).

As praias estuarinas estudadas na baía da Babitonga confirmaram sua importância como berçários para vários táxons de peixes, abrigando ao longo do ano abundâncias expressivas do ictioplâncton. Os resultados obtidos foram relevantes e mostraram a presença nas praias de fases iniciais de táxons que são representantes de: elos entre a base da cadeia trófica e os predadores de topo, estoques pesqueiros e espécie introduzida. Além disto, o enfoque pioneiro deste trabalho, realizado sobre um tipo de ambiente pouco estudado no Brasil, reforça a necessidade de mais pesquisas sobre ictioplâncton nas praias estuarinas, por exemplo, de interação com o padrão de circulação local, conectividade ou movimentos para o habitat adulto, crescimento, sobrevivência, assim como de geração de informações fomentadoras de planos de conservação.

\section{Agradecimentos}

Os autores agradecem o suporte da Universidade da Região de Joinville (UNIVILLE) e Universidade Federal do Paraná (UFPR). O auxilio em campo e laboratório de F.S. Döge, F.P. Camacho, A.M. Almeida, P.S.V. Pandolfo, L.N. Duarte, L.C.F.C. Silva, T. Soares, J.L.C. Serena, R.V.V. Navarro, P.C. Rocha, E. Melo, Sr. Tião, Sr. Valdir e Rubinho. O auxilio com a fluorimetria de L.R. Rörig e M.A. Castro-silva (UNIVALI). As críticas e sugestões de dois revisores anônimos que contribuíram para o aprimoramento deste trabalho.

\section{Referências}

BARLETTA-BERGAN, A.; BARLETTA, M.; SAINT-PAUL, U. Structure and seasonal dynamics of larval fish in the Caeté river estuary in North Brazil. Estuarine, Coastal and Shelf Science, London, v. 54, p. 193-206, 2002.

BECK, M. W.; HECK, K. L. Jr.; ABLE, K. W.; CHILDERS, D. L.; EGGLESTON, D. B.; GILLANDERS, B. M.; HALPERN, B.; HAYS, C. G.; HOSHINO, K.; MINELLO, T. J.; ORTH, R. J.; SHERIDAN, P. F.; WEINSTEIN, M. P. The identification, conservation, and management of estuarine and marine nurseries for fish and invertebrates. BioScience, Berkeley, v. 51, n. 8, p. 633-641, 2001.

BECK, M. W.; HECK, K. L. Jr.; ABLE, K. W.; CHILDERS, D. L.; EGGLESTON, D. B.; GILLANDERS, B. M.; HALPERN, B.; HAYS, C. G.; HOSHINO, K.; MINELLO, T. J.; ORTH, R. J.; SHERIDAN, P. F.; WEINSTEIN, M. P. The role of nearshore ecosystems as fish and shellfish nurseries. Issues in Ecology, Washington, v. 11, p. 1-12, 2003.

BOEHLERT, G. W.; MUNDY, B. C. Roles of behavioral and physical factors in larval and juvenile fish recruitment to estuarine nursery areas. American Fisheries Society Symposium, Bethesda, v. 3, p. 51-67, 1988. 
BONECKER, F. T.; CASTRO, M. S.; BONECKER, A. C. T. Larval fish assemblage in a tropical estuary in relation to tidal cycles, day/ night and seasonal variations. Pan-American Journal of Aquatic Sciences, Rio Grande, v. 4, n. 2, p. 238-245, 2009.

CHURCHILL, J. H.; FORWARD, R. B.; LUETTICH, R. A.; HENCH, J. L.; HETTLER, W. F.; CROWDER, L. B.; BLANTON, J. O. Circulation and larval fish transport within a tidally dominated estuary. Fisheries Oceanography, Cambridge, v. 8, p. 173-189, 1999.

CHUTE, A. S.; TURNER, J. T. Plankton studies in Buzzards Bay, Massachusetts, USA. V. Ichthyoplankton, 1987 to 1993. Marine Ecology Progress Series, Oldendorf, v. 224, p. 45-54, 2001.

CLAIREAUX, G.; AUDET, C. Seasonal changes in the hypoosmoregulatory ability of brook charr: the role of environmental factors. Journal of Fish Biology, Dumfries, v. 56, p. 347-373, 2000 .

CLARKE, K. R.; WARWICK, R. W. Change in marine communities: an aproach to statistical analysis and interpretation. [S.I.]: Plymouth: Plymouth Marine Laboratory. 2001. Disponível em: <www.pml.ac.uk/primer/>.

COSTA, M. D. P.; SOUZA-CONCEIÇÃO, J. M. Composição e abundância de ovos e larvas de peixes na baía da Babitonga, Santa Catarina, Brasil. Pan-American Journal of Aquatic Science, Rio Grande, v. 4, n. 3, p. 372-382, 2009.

COSTA, M. D. P.; SOUZA-CONCEIÇÃO, J. M.; SCHWINGEL, P. R.; SPACH, H. L. Assessment of larval distribution of invasive Omobranchus punctatus (Valenciennes, 1836) (Pisces: Blenniidae) in a subtropical estuary (Southern Brazil). Aquatic Invasions, Helsinki, v. 6, p. 33-38, 2011.

CREMER, M. J.; MORALES, P. R. D.; OLIVEIRA, T. M. N. Diagnóstico ambiental da baía da Babitonga. Joinville: UNIVILLE, 2006. 256 p.

ELLIOTT, M.; HEMINGWAY, K. L. Fishes in estuaries. Oxford: Blackwell Science Ltd., 2002. 636 p.

FAHAY, M. P. Guide to the early stages of marine fishes occurring in the western North Atlantic Ocean, Cape Hatteras to the Southern Scotian Shelf. Journal Northwest Atlantic Fisheries Science, Dartmouth, v. 4, p. 1-423, 1983.

FISHER, L. G.; PEREIRA, L. E. D.; VIEIRA, J. P. Peixes estuarinos e costeiros. Rio Grande: Ecoscientia, 2004. 127 p.

FREITAS, M. O.; VELASTIM, R. Ictiofauna associada a um cultivo de mexilhão Perna perna (Linnaeus, 1758) Norte Catarinense, Sul do Brasil. Acta Scientiarum Biological Sciences, Maringá, v. 32, n. 1, p. 31-37, 2010.

GARCIA, A. M.; VIEIRA, J. P. Abundância e diversidade da assembléia de peixes dentro e fora de uma pradaria de Ruppia maritima L., no estuário da Laguna dos Patos. Atlântica, Rio Grande, v. 19, p. 161-181, 1997.

GARCIA, A. M.; VIEIRA J. P. O aumento da diversidade de peixes no estuário da Lagoa dos Patos durante o episódio El Niño 19971998. Atlântica, Rio Grande, v. 23, p. 133-152, 2001.

GERHARDINGER, L. C.; FREITAS, M. O.; ANDRADE, A. B.; RANGEL, C. A. Omobranchus punctatus (Teleostei: Blenniidae), an exotic blenny in the Southwestern Atlantic. Biological Invasions, Dordrecht, v. 8, p. 941-946, 2006.
HAJISAMAE, S.; CHOU, L. M. Do shallow water habits of an impacted coastal strait serve as nursery grounds for fish? Estuarine, Coastal and Shelf Science, London, v. 53, p. 281-290, 2003.

HAKALA, T.; VIITASALO, M.; RITA, H.; ARO, E.; FLINKMAN, J.; VUORINEN, I. Temporal and spatial variation in the growth rates of baltic herring (Clupea harengus membras L.) larvae during summer. Marine Biology, Kiel, v. 142, p. 25-33, 2003.

HOUDE, E. D.; ZASTROW, C. E. Ecosystem and taxon-specific dynamic and energetics proprieties of larval fish assemblages. Bulletin of Marine Science, Miami, v. 53, n. 2, p. 290-335, 1993.

IBAMA. Proteção e controle de ecossistemas costeiros Manguezal da Baía da Babitonga. Série Estudos da Pesca n 25. Brasília: IBAMA, 1998. 147 p.

IKEJIMA, K.; TONGNUNUI, P.; MEDEJ, T.; TANIUCHI, T. Juvenile and small fishes in a mangrove estuary in Trang province, Thailand: seasonal and habitat differences. Estuarine, Coastal and Shelf Science, London, v. 56, p. 447-457, 2003.

JACKSON, N. L.; NORDSTROM, K. F.; SMITH, D. R. Geomorphic-biotic interactions on beach foreshores in estuaries. Journal of Coastal Research, Fort Lauderdale, v. 36, p. 414-424, 2002.

JOBLING, M. Environmental biology of fishes. London: Chapman \& Hall, 1996. 455 p.

JOHANNES, R.E. Reproductive strategies of coastal marine fishes in the tropics. Environmental Biology of Fishes, New York, v. 3, n. 1, p. 65-84, 1978.

KAWAGUCHI, T.; KOHNO, H.; FUJITA, K.; TAKI, Y. Early morphological development of Omobranchus fasciolatoceps and O. punctatus (Blenniidae: Omobranchini) reared in an aquarium. Ichthyological Research, Tokyo, v. 46, n. 2, p. 163-170, 1999.

KNIE, J. L. W. Atlas ambiental da região de Joinville: complexo hídrico da bBaía da Babitonga. Joinville: FATMA/GTZ, 2002. 144 p.

LASKER, R. Marine fish larvae: morphology, ecology, and relation to fisheries. Washington: Washington Sea Grant Program, 1984. $131 \mathrm{p}$.

LEIS, J. M.; RENNIS, D. S. The larvae of Indo-Pacific coral reef fishes. Hawai: University of Hawai Press, 1983. 269 p.

LEIS, J. M.; TRNSKI, T. The larvae of Indo-Pacific Shorefishes. Hawai: University of Hawai Press, 1989. 371 p.

LORENZ, J. The response of fishes to physicochemical changes in the Mangroves of northeast Florida Bay. Estuaries, New York, v. 2B, p. 500-517, 1999.

LUGENDO, B. R.; NAGELKERKEN, I.; JIDDAWI, N.; MGAYA, Y. D.; VAN DER VELDE, G. Fish community composition of a tropical nonestuarine embayment in Zanzibar, Tanzania. Fisheries Science, Amsterdam, v. 73, p. 1213-1223, 2007.

MACEDO-SOARES, L. C. P.; BIROLO, A. B.; FREIRE, A. S. Spatial and temporal distribution of fish eggs and larvae in a subtropical coastal lagoon, Santa Catarina State, Brazil. Neotropical Ichthyology, Porto Alegre, v. 7, n. 2, p. 231-240, 2009.

MACHADO, E. C.; DANIEL, C. B.; BRANDINI, N.; QUEIROZ, R. L. V. Temporal and spatial dynamics of nutrients and particulate suspended matter in Paranaguá Bay, PR, Brazil. Nerítica, Curitiba, v. 11, p. 15-34, 1997. 
MARMIL. Porto de São Francisco do Sul. Capitania dos Portos de Santa Catarina. 2012. Disponível em: <https://www.mar.mil.br/ cpsc/>. Acesso em: 31 jan. 2012.

McLUSKY, D. S. The estuarine ecosystem. New York: Chapman \& Hall, 1994. 215 p.

MOSER, H. G. The early stages of fishes in the California Current Region. California Cooperative Ocean Fisheries Investigations (CALCOFI). ATLAS n. 33. Lawrence: Allen Press Inc., 1996. $1505 \mathrm{p}$.

MUELBERT, J. H.; WEISS, G. Abundance and distribution of fish larvae in the channel area of the Patos Lagoon Estuary, Brazil. Proceedings of the annual fish conference: Larval fish recruitment and research in the Americas, Merida, v. 13, p. 4354, 1991.

RÉ, P. M. A. B. Ictioplâncton estuarino da península Ibérica (Guia de identificação de ovos e estados larvares planctônicos). Lisboa: Universidade de Lisboa, 1999. 78 p.

RICHARDS, W. J. Early stages of Atlantic fishes. An identification guide for the Western Central North Atlantic. Boca Raton: CRC / Taylor \& Francis, 2006. 2640 p.

RÖNNBÄCK, P. The ecological basis for economic value of seafood production supported by mangroves ecosystems. Ecological Economics, Amsterdam, v. 29, p. 235-252, 1999.

ROUNTREE, R. A.; ABLE, K. W. Fauna of polyhaline subtidal marsh creeks in southern New Jersey: composition, abundance and biomass. Estuaries, New York, v. 15, p. 171-185, 1992.

SINQUE, C.; MUELBERT, J. H. Ictioplâncton. In: SEELIGER, U.; ODEBRECHT, C.; CASTELLO, J. P. (Eds). Os Ecossistemas costeiro e marinho do extremo sul do Brasil. Rio Grande: Ecoscientia, 1998. p. 56-60.
SOKAL, R. R.; ROHLF, F. J. Biometry: the principles and practices of statistics in biological. research. New York: W. H. Freeman and Co., 1995.887 p.

SOUZA-CONCEIÇÃO, J. M.; RODRIGUES-RIBEIRO, M.; CASTRO-SILVA, M. A. Dinâmica populacional, biologia reprodutiva e o ictioplâncton de Cetengraulis edentulus Cuvier (Pisces, Clupeiformes, Engraulidae) na enseada do saco dos Limões, Florianópolis, Santa Catarina, Brasil. Revista Brasileira de Zoologia, Curitiba, v. 22, n. 4, p. 953-961, 2005.

THAYER, G. W.; POWELL, A. B.; HOSS, D. E. Composition of larval, juvenile, and small adult fishes relative to changes in environmental conditions in Florida Bay. Estuaries, New York, v. 22, n. 2B, p. 518-533, 1999.

TSUZUKI, M. Y.; AIKAWA, H.; STRÜSSMANN, C. A.; TAKASHIMA, F. Physiological responses to salinity increases in the freshwater silversides Odontesthes bonariensis and O. hatchery (Pisces, atherinidae). Revista Brasileira de Oceanografia, São Paulo, v. 48, n. 1, p. 81-85, 2000.

VÉLEZ, J. A.; WATSON, W.; ARNTZ, W.; WOLFF, M.; SCHNACK-SCHIEL, S. B. Larval fish assemblages in Independencia Bay, Pisco, Peru: temporal and spatial relationships. Marine Biology, Kiel, v. 147, p. 77-91, 2005.

WEISBERG, S. B.; WILSON, H. T.; HIMCHAK, P.; ALLEN, R. Temporal trends in abundance of fish in the tidal Delaware river. Estuaries, New York, v. 19, n. 3, p. 723-729, 1996.

WOOTTON, R. J. Fish ecology. New York: Chapman and Hall, 1992. $212 \mathrm{p}$. 